\title{
A Dimmable Light-Emitting Diode (LED) Driver With Mag-Amp Postregulators for Multistring Applications
}

\author{
Wu Chen, Student Member, IEEE, and S. Y. R. Hui, Fellow, IEEE
}

\begin{abstract}
Current imbalance should be avoided when multiple LED strings are connected in parallel. In this paper, a dimmable LED driver with magnetic-amplifier postregulators for multistring applications is presented. Powered by a common master source, parallel LED strings are individually regulated by their corresponding adaptive slave sources for current balancing in this proposal. Without linear current regulators, the proposed driver offers relatively high efficiency. Its structure is simpler than multiconverter structures for red, blue, and green LED applications, and is particularly suitable for LEDs with wide parameter variations. The performance of the proposed driver is experimentally verified by a 16.5-W prototype with a load of three 5.5-W LED strings.
\end{abstract}

Index Terms-Current sharing, light-emitting diode (LED), magnetic amplifier.

\section{INTRODUCTION}

$\mathbf{R}$ ECENTLY, LEDs have been applied to liquid crystal display (LCD) backlight or display panel, streetlights, signage, and general-purpose lighting due to the rapid progress achieved in the solid-state lighting technology. Compared with existing conventional lighting sources such as energyinefficient incandescent lamps and mercury-based fluorescent lamps, LEDs have relatively longer lifetime in the range of 80000-100000 h [1]. Meanwhile, LEDs available in the market are encapsulated with less glass, which significatly improves their reliability and safety to the handler. Besides, mercuryfree LEDs are environmentally friendly and can be disposed safely at the end of their lifetime. LEDs also have flicker-free, smooth-dimming, low-voltage operation, and good color rendering properties [2]-[4].

Presently, the power ratings of individual LED devices are a few watts, limited by the packaging technology and heat dissipation. To obtain sufficient luminance for some high-power applications, such as streetlight and large-scale LCD panels,

Manuscript received May 14, 2010; revised July 12, 2010; accepted August 25, 2010. Date of current version July 22, 2011. Recommended for publication by Associate Editor M. Ponce-Silva.

W. Chen is with the Aero-Power Sci-Tech Center, College of Automation Engineering, Nanjing University of Aeronautics \& Astronautics, China (e-mail: chenwu@nuaa.edu.cn).

S. Y. R. Hui is with the Centre for Power Electronics, City University of Hong Kong, Hong Kong, China, and also with Imperial College London, London SW7 2AZ, U.K. (e-mail: eeronhui@ cityu.edu.hk).

Color versions of one or more of the figures in this paper are available online at http://ieeexplore.ieee.org.

Digital Object Identifier 10.1109/TPEL.2010.2082565 many LEDs are connected and arranged in parallel LED strings. The general photoelectrothermal (PET) theory also indicates that a distributed LED system based on a plurality of relatively low-power LEDs can have advantages over a concentrated system consisting of a small number of high-power LEDs for the same system power [5], [6]. Therefore, using LED strings in parallel has been a commom practice. The use of parallel LED strings inevitably leads to current imbalance problem due to the LED parameter variations, aging, and temperature changes, which will in turn affect the luminous intensity and even color in each string [7]. Most importantly, if the current imbalance causes one or more LED strings to exceed their rated current values, the lifetime of the LED strings (and hence, the LED system) will be drastically reduced.

Several current sharing methods for driving multistring LED systems have been previously proposed. The simplest approach is to add a ballast resistor in series with each LED string in order to minimize the current imbalance [8]. Capacitor can be used to replace the dissipative ballast resistor to reduce the resistive loss when the LEDs are driven by ac source or coupled with rectifier [9]-[11]. The main drawback of these methods is that the forward current of each LED string cannot be precisely controlled. Another approach is to employ a linear current regulator for each string [12]-[19]. An alternative method is to set up an individual voltage source for each LED string [20]-[22] to regulate the current at the expense of increased circuit complexity and costs. In [21] and [22], two modular power converter architectures based on parallel or series-input-connected converters with separate LED string loads have been suggested. Each LED string current is separately sensed and controlled for current matching. Without dissipative ballast resistor or linear current regulator, these two LED driver architectures have relatively higher conversion efficiency. However, the architectures are complex and expensive because each LED string needs a set of main power circuit and controller. In [23], an effective twosecondary-output-windings structure was proposed for multiple LED strings with independent brightness control. Although the LED driver with two-secondary-output-windings structure is relatively simpler and cheaper than the modular converter architectures, it still has some limitations. For example, it is only suitable for constant input voltage (the constant output of first-stage power factor correction regulator is supplied for the second-stage converter) and separate IC controller is needed for each LED string.

A dimmable driver for multiple LED strings is proposed in this paper to overcome the aforementioned limitations. With one 


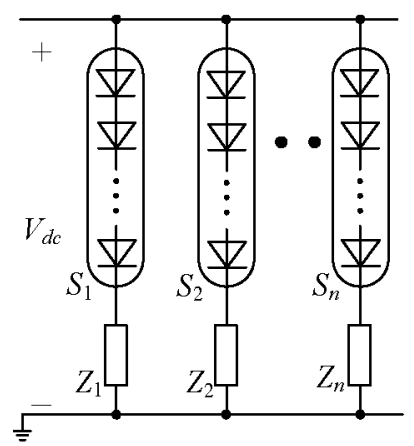

(a)

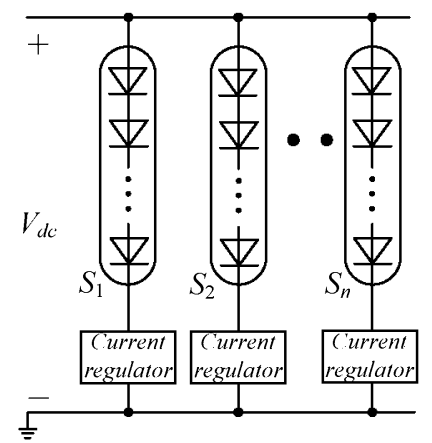

(b)

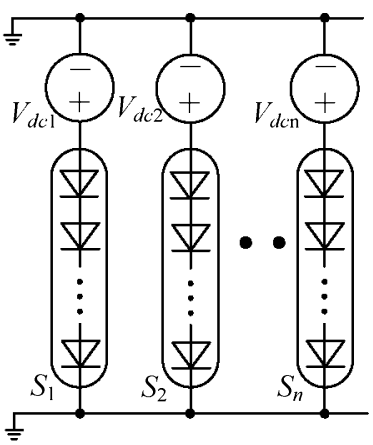

(c)

Fig. 1. Three approaches for reducing current imbalance in parallel LED strings. (a) Series impedance. (b) Series linear current regulator. (c) Dedicated dc/dc converter.

common bulk dc power supply for all LED strings, the proposed driver employs a magnetic-amplifier (mag-amp) postregulator as a partial and adaptive voltage for regulating the current in each LED string. Since all LED strings share one common majority power supply and the mag-amp postregulator handles only a fraction of the LED power, the power ratings of the mag-amp postregulators are small and the overall cost of the system is reasonable for high-performance LED systems with good current balance in parallel LED strings.

\section{Overview of Existing CurRent Sharing Methods}

In this section, several current balance methods are briefly reviewed. They are divided into three categories: 1) series impedance method; 2) series linear-current-regulator method; and 3) dedicated dc/dc converter method [11].

\section{A. Series Impedance Method}

Each LED string can be regarded as an equivalent dynamic resistive load with its value equal to the ratio of the string voltage to the string current. The extent of current imbalance among parallel strings depends on the variation or mismatch of their equivalent resistances. The simplest form of series impedance method is to add a series resistor of identical resistance in each LED string, as shown in Fig. 1 (a). If the current of a particular string is higher than the others, then the voltage drop across this series resistor increases. Consequently, the voltage drop across the LED string will be reduced, resulting in a reduction of string current. However, for effective reduction in current imbalance, the value of the series resistor has to be sufficiently large (e.g., series resistors of $60 \Omega$ are used in [8]). To avoid power loss introduced by the series resistor, lossless capacitor can be connected in series with each antiparallel LED strings driven by an ac source [9], [10]. However, each LED string conducts only half of each mains cycle and such a utilization rate of LEDs is relatively poor. To overcome this drawback, Choi et al. [11] proposed a symmetric current balance circuit, in which half-wave rectifiers are used to provide dc source for each LED string. The main drawback of these series impedance approaches is that the forward current of each LED string cannot be precisely controlled. They may suit some applications, which do not need strict requirement for current balance, such as street lighting, but may not be suitable for some applications, such as display, that require tight current control.

\section{B. Series Linear-Current-Regulator Method}

In order to precisely control each LED string current, a linear current regulator can be used in each string, as shown in Fig. 1 (b). The LED string current is sensed and regulated with closed-loop control. The power losses on the linear current regulators are considerable because the voltage $V_{d c}$ has to be high enough to maintain all the strings at the desired current under all conditions. In [12] and [13], a method was proposed to minimize the power losses on the current regulators. The lowest voltage drop across the current regulators is sensed to determine the proper $V_{d c}$ for LED strings through feedback control. An improved method was proposed in [14] to avoid the temperature dependence of the forward voltage drop of the sensing diodes and nonoptimal reference voltage, and hence, the conversion efficiency can be improved. With an operating principle similar to that of linear regulators, current mirror circuits can also be applied to multiple LED strings application to reduce current imbalance [19]. Although the improved efficiency can be achieved [14], the linear-current-regulator method still dissipates considerable power.

\section{Dedicated DC/DC Converter Method}

The voltage across each LED string can be provided with separate dc source to eliminate the power dissipation on the current regulator or current mirror circuits, as shown in Fig. 1 (c). In [20], three dc/dc converters are provided for red, green, and blue (RGB) LED strings, respectively. In [21], six synchronous rectifier Buck converters are built up for six LED strings, respectively, and each string current is sensed and controlled independently to the same level. For high input voltage applications, $\mathrm{Hu}$ and Zane [22] proposed a modular converter architecture based on series-input-connected converter cells, which independently drive the LED strings. These driver systems are relatively expensive because each LED string needs a separate power converter. In [24], a multioutput flyback converter is designed to provide three output voltages for RGB LED strings, respectively. In [25], 


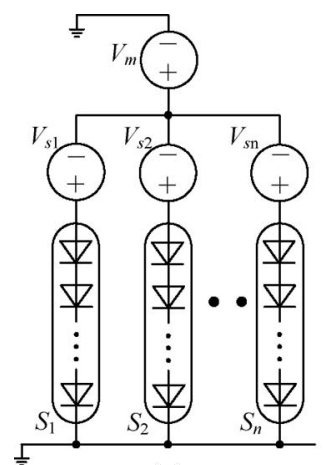

(a)

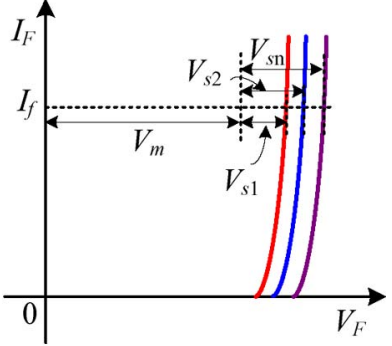

(b)
Fig. 2. Block diagram of the proposed LED driver.

a multioutput converter with current ripple-free topology based on mag-amp postregulator is proposed. This interesting idea utilizes the parallel master and slave converter concept for multistring application, and the use of passive ripple cancellation to provide smooth current control for LED strings. The master and slave converters are all electrically isolated from one another. In [23], a two-secondary-output-windings structure is proposed for multiple LED strings with independent brightness control. Compared with multiconverter systems, the multioutput converters have simpler structures. However, they still suffer from some limitations such as output voltages cannot be directly regulated to achieve maximum conversion efficiency and/or a separate IC controller is needed for each LED string.

\section{Proposed LED Driver With Master-Slave STRUCTURE}

The power handling principle of the proposal is illustrated in Fig. 2. Unlike the fully isolated master and slave sources approach in [25], the proposed LED driver involves a "common" master voltage source $V_{m}$ for all LED strings and a separate slave voltage source $\left(V_{s 1}-V_{s n}\right)$ for each LED string for current regulation, as shown in Fig. 2 (a). The majority of power consumed in each string is fed by the "master" voltage source, while the corresponding slave voltage source is used to regulate the current for current balancing. To avoid large power losses, all the master and slave dc sources should be switch-mode converters. This is very important for LEDs with wide device parameter tolerances. For example, in [12], the forward voltages of eight LED strings at $45 \mathrm{~mA}$ vary from 21.9 to $31.7 \mathrm{~V}$. If linear current regulators are used, the voltage drop across the linear current regulator of the string with minimum forward voltage will be up to $9.8 \mathrm{~V}$, and the power loss in the current regulator is unacceptable if the forward current is large. For example, a typical string current of $0.3 \mathrm{~A}$ will lead to about power loss of $3 \mathrm{~W}$ in each string. With the proposed LED driver structure, a $20-\mathrm{V}$ master source common for all eight strings and a separate low-voltage slave voltage source covering the voltage difference among the parallel LED strings can be constructed in each string. Thus, the majority of the power is provided by the master source and only the remaining power is provided by the corresponding slave source.

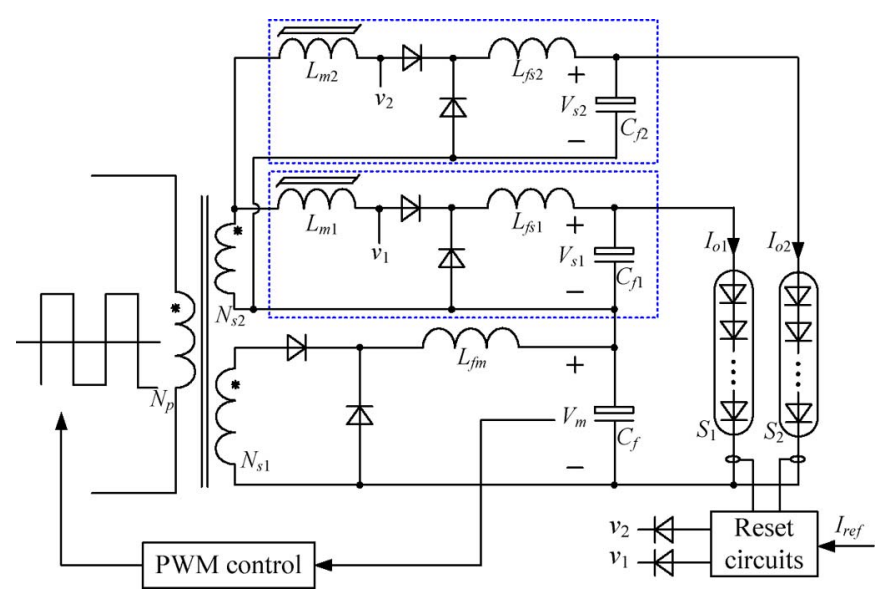

Fig. 3. Circuit diagram of the proposed LED driver.

Various topologies with independent multiple outputs have been previously proposed. Examples are the use of postregulators in the transformer secondary sides with mag-amp [26] or synchronous switch [27], multiple-winding flyback converters [28], and pulse width modulation (PWM)-pulse delay (PWM-PD) control method [29]. These methods have their own features and specific applications.

For the proposed LED driver with multiple outputs, some technical requirements have to be considered.

1) Electrical Isolation: Electrical isolation is necessary between the master source and the slave sources because their terminals cannot share the same ground. However, it is not necessary to carry out electrical isolation among the slave sources.

2) Regulated Outputs: The voltage across the LED string must be regulated to adapt to different forward currents and ambient temperature. Hence, independently and precisely regulated multiple outputs are required.

3) Modularity: It is required that the topology is easily expanded and therefore a modular approach is preferred.

4) Power Distribution: The majority of the LED power should be provided by the master source and the remaining power provided by the slave sources. The circuit implementation should achieve such power distribution.

Based on these considerations, the proposed LED driver topology with mag-amp postregulators in Fig. 3 is adopted. The mag-amp postregulator topology has been used in the highperformance power supplies with multiple outputs because of their high efficiency, high stability, high power density, simple control, and low electromagnetic interference [30]. In our proposal, only two secondary windings of the transformer are used to generate a plurality of outputs. One secondary winding output is used for the master source $V_{m}$, which is PWM controlled by a power converter on the primary side. The other secondary winding output is used to generate multiple slave sources $V_{s 1}$, $V_{s 2}, \ldots, V_{s n}$ (only two slave sources are shown in Fig. 3) based on separate mag-amp regulators with feedback loop through sensing LED forward current. It is worthwhile to note that the differences between the proposed structure in the paper and the 
one presented in [25]. First, only two secondary windings are needed in this proposal to generate multiple outputs, thus resulting in simpler transformer structure, lower production cost, and less leakage inductance. Second, each mag-amp regulator is used to handle only a small portion of the power in each LED string. Therefore, the size of the mag-amp core is much smaller and its power loss is much less than those in [25].

\section{ANALYSIS AND DESIGN CONSIDERATIONS}

The mag-amp regulators provide power regulation functions for a portion of the power in each LED string. If the string current $I_{o 1}$ is larger than a reference current $I_{r e f}$, then the duration of the blocking time of the mag-amp inductor $L_{m 1}$ will be increased by adjusting the output of the reset circuit, leading to the decline of the $V_{s 1}$, and the subsequent reduction of $I_{o 1}$ to follow $I_{r e f}$. As shown in Fig. 3, it can be seen that the power provided for each LED string is composed of two parts, the master source and the separate slave source. If the forward voltage drop of a LED string is constant, there are countless distribution combinations between the master source and the slave one. Hence, determining how to find the optimal distribution will be qualitatively analyzed here.

\section{A. Power Distribution}

First, the voltage of the master source must be lower than all the forward voltage drops of the LED strings under whole operating conditions because the master source should provide the majority (but not all) of the power for all the LED strings, i.e., $V_{m}<V_{\text {led_min }}$. The slave sources should be able to adjust the part of the voltage across LED strings to regulate their forward currents.

Second, the lower the master source is, the higher the slave source becomes, because the sum of them should be equal to the voltage across the entire LED string. In the extreme case, if the voltage of the master source is equal to zero, the proposed circuit reduces to the conventional converter with multiple outputs, in which case each LED string is fully powered by a single source, as described in [25]. Two disadvantages arise from this extreme case. The voltage stress of the rectifier diodes in each source will be increased significantly and therefore high-voltage diodes with relatively high voltage drop have to be used. The second disadvantage is that a larger output filter inductor will be needed in each source to meet the output current ripple requirement. Besides, the power losses on the mag-amp will be increased because it has to block a voltage high enough for the entire LED string. In this proposal, the common power supply provides the main voltage for all LED strings. Thus, power diodes with low voltage ratings and low forward voltage drop such as Schottky diodes and smaller output filter inductors can be chosen for the slave source.

In this analysis, we assume that all the LED strings share the same current and have the same forward voltage drop $V_{F}$, and the sum of all string currents is $I_{F}$. All the output filter inductor currents are assumed to have the same ripple current factor $\gamma$. For the structure presented in [25], the required output filter

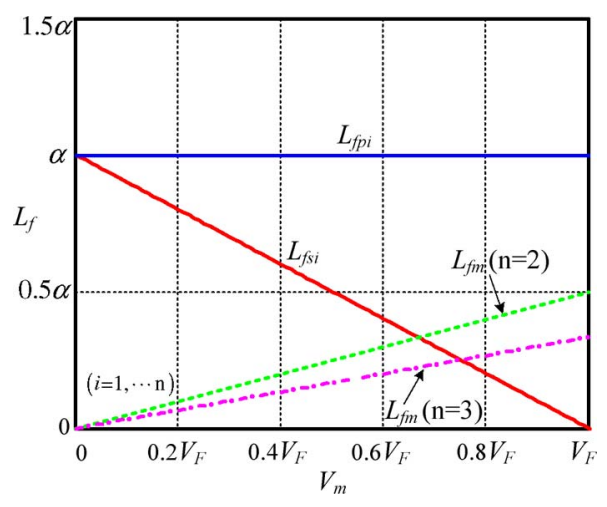

Fig. 4. Curves of $L_{f}$ versus $V_{m}$.

inductor in each output is

$L_{f p i}=\frac{V_{F}(1-D) T_{s}}{\gamma \cdot I_{F} / \mathrm{n}}=\frac{V_{F}(1-D) T_{s}}{\Delta I_{F} \text { slave }}=\alpha \quad(i=1, \ldots, n)$

where $D$ is the duty cycle of the voltage pulse in secondary winding, $T_{s}$ is the switching period of the voltage pulse in secondary winding, and $n$ is the number of LED strings.

For convenience, the value of $V_{F}(1-D) T_{s} / \Delta I_{F_{\text {slave }}}$ in (1) is defined as $\alpha$.

Then, for the proposed driver, the required output filter inductor for the master source is

$$
L_{f m}=\frac{V_{m}(1-D) T_{s}}{\gamma I_{F}}=\frac{V_{m}}{n \cdot V_{F}} \cdot \alpha
$$

The required output filter inductor in each slave source is

$$
\begin{aligned}
L_{f s i} & =\frac{\left(V_{F}-V_{m}\right)(1-D) T_{s}}{\gamma \cdot I_{F} / \mathrm{n}} \\
& =\frac{V_{F}-V_{m}}{V_{F}} \cdot \alpha \quad(i=1, \ldots, n) .
\end{aligned}
$$

Then, we can obtain the curves of $L_{f}$ versus $V_{m}$, as shown in Fig. 4. If the load has three LED strings, i.e., $n=3$, if we set $V_{m}=0.9 V_{F}$, (i.e. the common power supply provides $90 \%$ of the output voltage), then one inductor for $V_{m}$ with $0.3 \alpha$ and three inductors for $V_{s 1}-V_{s 3}$ with $0.1 \alpha$ each are needed in the proposed driver. These inductor requirements are compared with three output filter inductors with $\alpha$ each in the structure presented in [25].

Based on the core loss formula in [35], the core loss in the mag-amp core is

$$
P_{\text {core }}=\left(9.93 \times 10^{-6}\right) \cdot\left(f^{1.57}\right) \cdot\left(B^{1.70}\right) .
$$

Equation (4) indicates that the core loss is proportional to the magnetic flux density. In our proposal, the voltage of the slave source that uses the mag-amp core is much lower than the full voltage across the LED string. Therefore, (4) theoretically confirms that mag-amp power loss in this proposal is also reduced. 


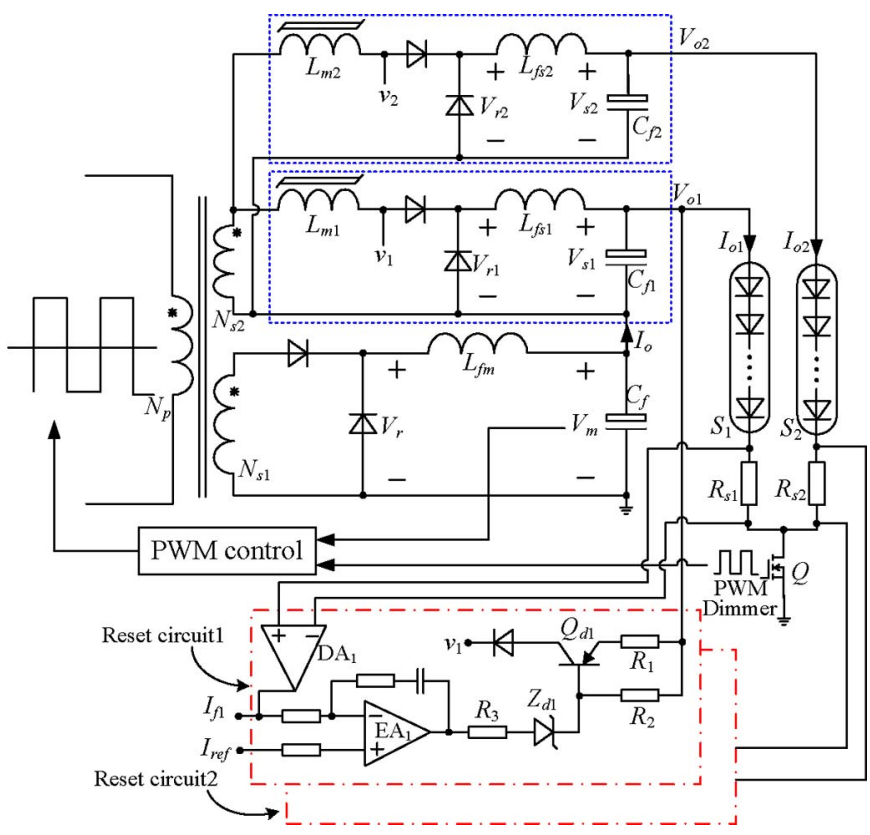

Fig. 5. Proposed LED driver with conventional PWM dimming.

\section{B. Dimming Methods}

Traditionally, there are two kinds of dimming techniques for driving LEDs: amplitude mode and PWM mode. The main characteristics and differences between the two modes can be found in [31] and [32]. However, PWM dimming methods have been better received for high-performance applications such as display panels because the current level and hence the color temperature of the LED can be maintained, although the amplitude mode is acceptable for general public lighting applications.

In the proposal, dimming can be achieved through conventional PWM scheme and a phase-shift PWM (PSPWM) scheme. The circuit diagram of the proposed LED driver system with conventional PWM dimming function is shown in Fig. 5 (only two LED strings are shown), in which $R_{s 1}$ and $R_{s 2}$ are the current sensing resistors for LED string $S_{1}$ and $S_{2}$, respectively, $Q$ is the PWM dimming switch, and the circuit inside the dotted box is the reset circuit for mag-amp. Unlike the conventional PWM dimming method used with linear current regulators [15], only one MOSFET (dimming switch) is needed for all LED strings in this proposal and it is operated not in the linear ohmic region, but in the saturation region. Hence, the conduction power losses in the dimming process can be reduced. However, differential amplifiers $\left(D_{1}\right)$ are needed to sense the LED current signals because all the current sensing resistors do not share the common ground (as only one dimming switch $Q$ is used). The sensed current signal is compared with $I_{r e f}$ to regulate the reset current of the mag-amp. The special use of the zener diode $Z_{d 1}$ is to act as a voltage level shifter because the multiple output voltages of the converter may not be the same voltage level of the error amplifier. During the time interval when $Q$ is tuned off, the highfrequency switching operation of the primary main switches can be disabled to further reduce the switching loss. If the dimming switch $Q$ is shorted, then the amplitude-mode dimming can be achieved by regulating the current reference $I_{\text {ref }}$.

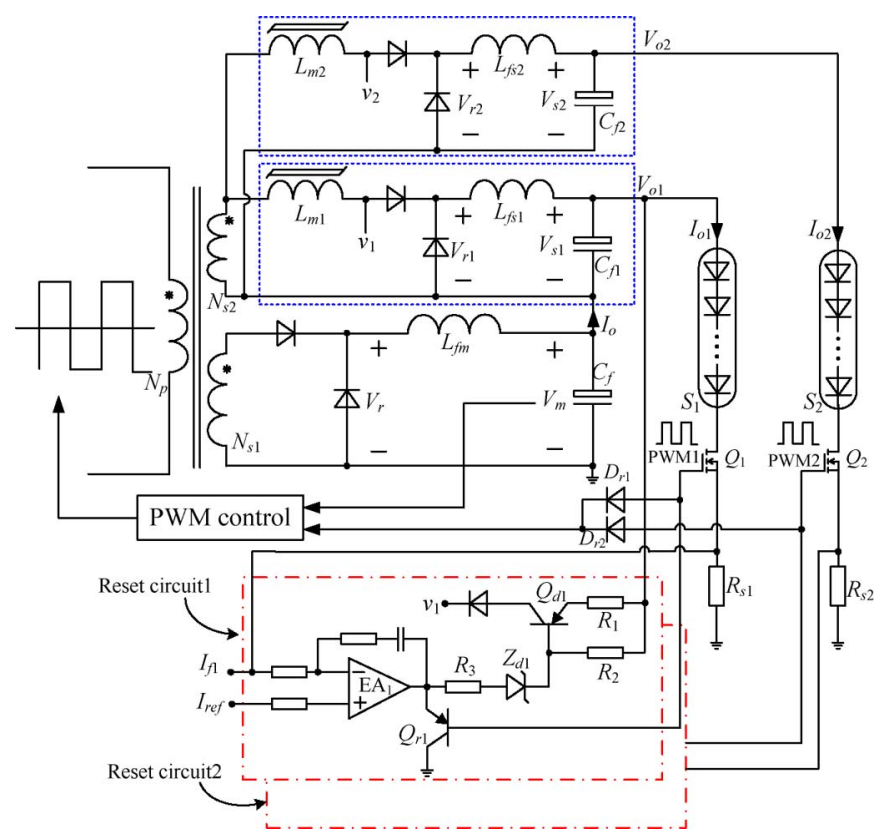

Fig. 6. Proposed LED driver with PSPWM dimming.

TABLE I

KEY COMPONENTS LIST

\begin{tabular}{|c|c|}
\hline Primary main switch & IRF540N \\
\hline Turns ratio of transformer & $N_{p}: N_{r}: N_{s 1}: N_{s 2}=12: 12: 24: 5$ \\
\hline Rectifier of master source & BYW51-200 \\
\hline Rectifier of slave source & $42 \mathrm{CTQ} 030 \mathrm{~S}$ \\
\hline Filter inductor of master source & $530 \mu \mathrm{H}$ \\
\hline Filter inductor of slave source & $170 \mu \mathrm{H}$ \\
\hline PWM controller & SG3525A (120 kHz) \\
\hline$L_{m 1} \sim L_{m 3}$ & AMG-12S, 16T \\
\hline$Z_{d 1} \sim Z_{d 3}$ & $1 \mathrm{~N} 5349 \mathrm{~B}$ \\
\hline$Q_{d 1} \sim Q_{d 3}$ & TIP127 \\
\hline $\mathrm{EA}_{1} \sim \mathrm{EA}_{3}$ & $\mathrm{LM} 358$ \\
\hline
\end{tabular}

To avoid the drawback of the conventional PWM dimming such as large pulsating input/output current and degraded EMI performance, PSPWM dimming function can be adopted [33], [34]. The proposed LED driver system with PSPWM dimming function is shown in Fig. 6, in which one dimming switch is used for each LED string. In the reset circuit of Fig. 6, a PNP transistor $\left(Q_{r 1}\right)$ is added to the output of the error amplifier. Its role is explained as follows. Taking LED string $S_{1}$ as an example. During the time interval when $Q_{1}$ is turned off, the sensed current is zero and the output voltage of $\mathrm{EA}_{1}$ is high if no $Q_{r 1}$ is used. This situation will result in a reset current 


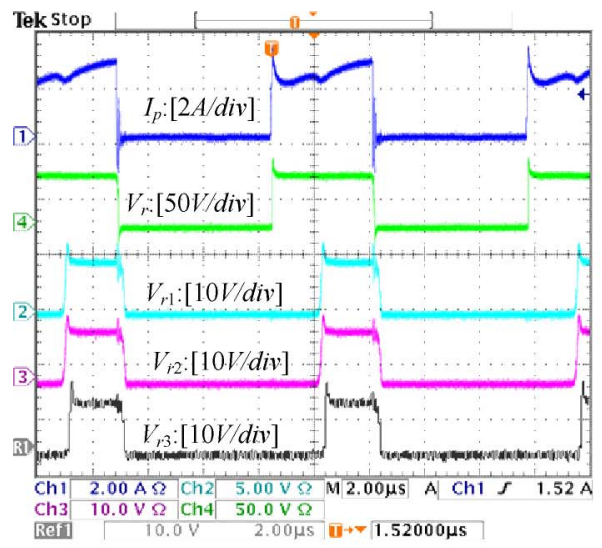

(a)

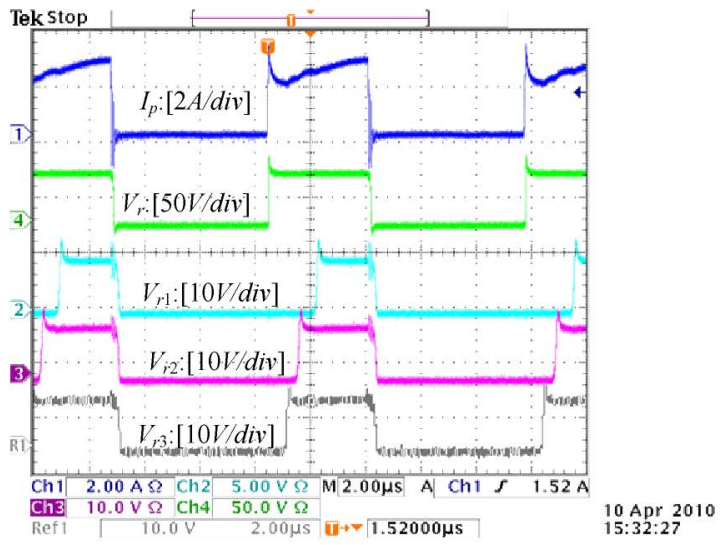

(b)

Fig. 7. Waveforms of the primary switch current $I_{p}$ and secondary rectifier voltages $V_{r}, V_{r 1}, V_{r 2}$, and $V_{r 3}$. (a) Matched LED strings and (b) unmatched LED strings.

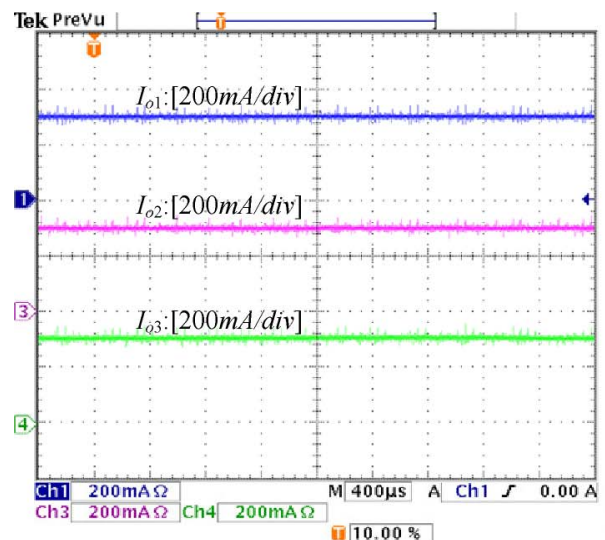

(a)

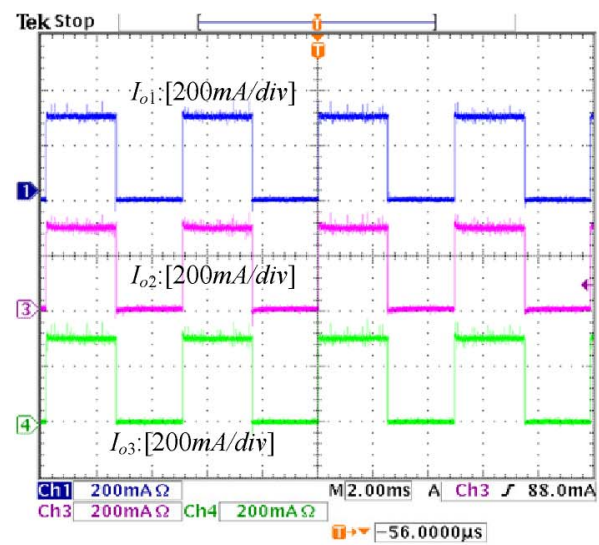

(c)

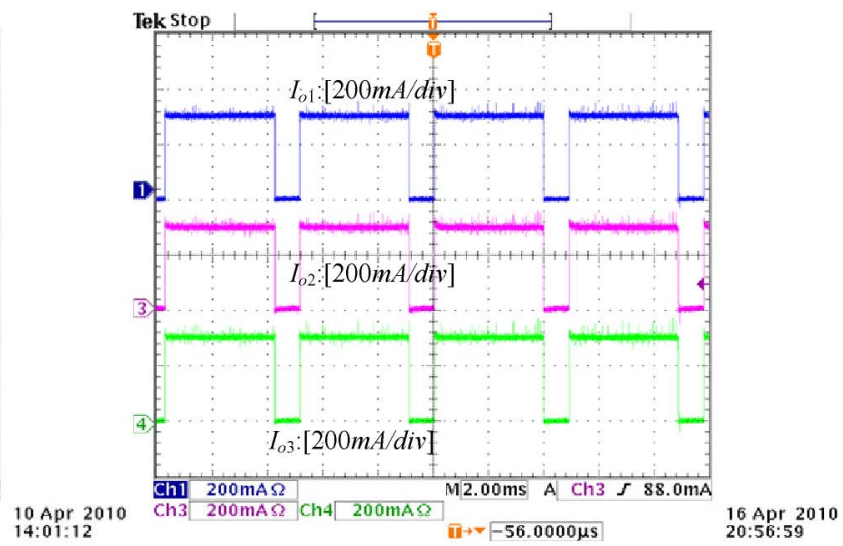

(b)

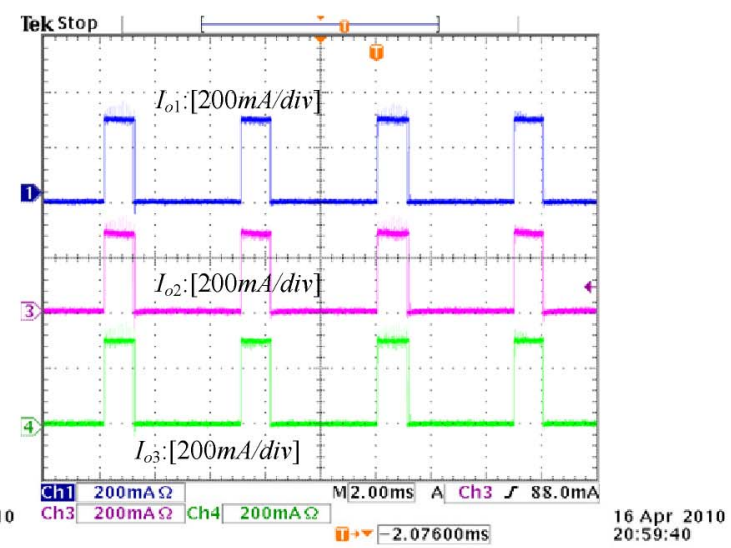

(d)

Fig. 8. Measured LED string current waveforms under (a) 100, (b) 80, (c) 50, and (d) 20\% conventional PWM dimming operations.

too small for the saturable reactor $L_{m 1}$, and $L_{m 1}$ will lose its mag-amp function and $V_{s 1}$ will rise far from its desired value, i.e., $V_{s 1}$ is out of control. When $Q_{r 1}$ is added, the output voltage of $\mathrm{EA}_{1}$ is almost zero, and then, the reset current for $L_{m 1}$ will increase to block the voltage pulse from the secondary winding $N_{s 2}$. Hence, the saturable reactor must be designed to have the ability to withstand the entire volt-second product of the input waveform. The design procedures for the saturable reactor and the reset circuit can be found in [35] and [36]. In the case, where all the PWM dimming signals are simultaneously low, the main switches in the primary side of the transformer can be turned off to reduce the switching losses. All the PWM dimming signals are OR-ed via $D_{r 1}$ through $D_{r n}$ to detect the signal. 


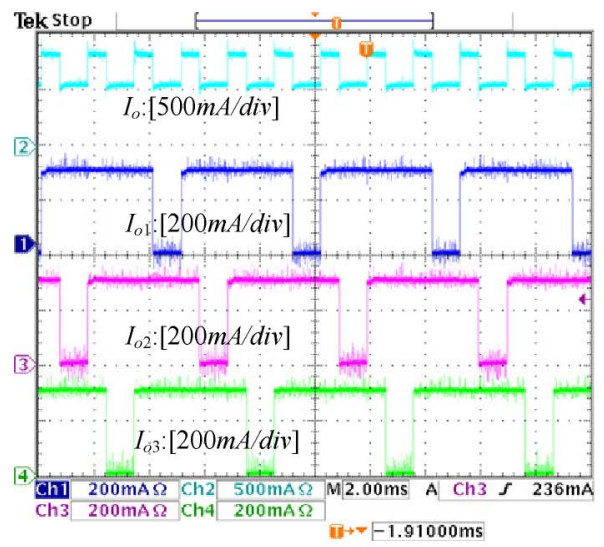

(a)

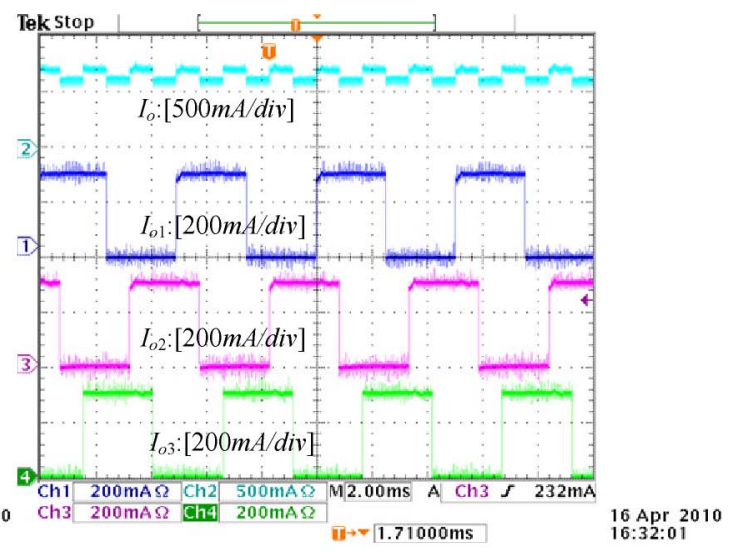

(b)

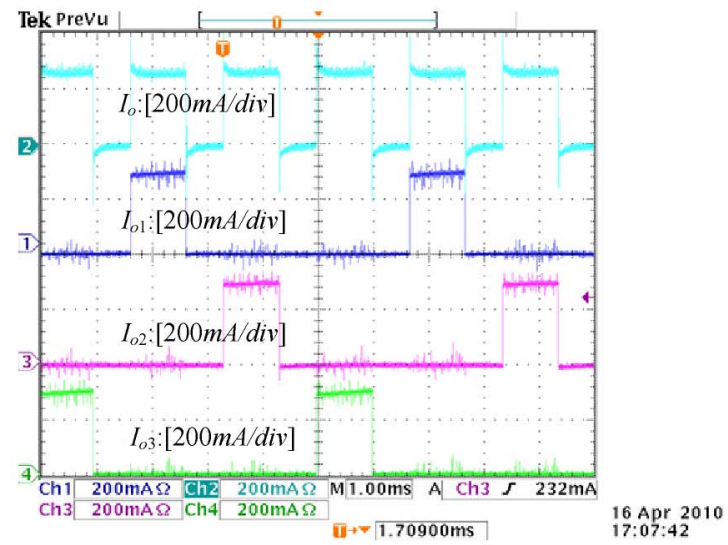

(c)

Fig. 9. Measured LED string current waveforms under (a) 80, (b) 50, and (c) 20\% PSPWM dimming operations.

\section{EXPERIMENTAL RESULTS}

The performance of the proposed LED driver was verified by a prototype with a $120-\mathrm{kHz}$ single-ended forward converter with tertiary transformer reset winding operating from a voltage source in 20-30 V. Three parallel strings of CREE cool white LEDs (model number: XREWHT-L1-WG-Q5-0-04) with six LEDs connected in series in each string are used to evaluate the performance of the proposed LED driver. The typical forward voltage of each LED is $3.3 \mathrm{~V}$ with $350 \mathrm{~mA}$, and the desired $V_{m}$ is set as $17 \mathrm{~V}$. The key components of the circuit are listed in Table I. The inductor values are determined by (1)-(3).

Fig. 7 shows the key waveforms of the LED driver. Fig. 7 (a) shows the waveforms of the primary switch current $I p$ and secondary rectifier voltages $V_{r}, V_{r 1}, V_{r 2}$, and $V_{r 3}$. It can be seen that the pulse widths are not identical. The pulse width of $V_{r 3}$ is slightly shorter than that of $V_{r 1}$ and $V_{r 2}$. The measured voltages are $V_{m}=17.06 \mathrm{~V}, V_{s 1}=1.87 \mathrm{~V}, V_{s 2}=1.88 \mathrm{~V}$, and $V_{s 3}=1.72 \mathrm{~V}$. In order to demonstrate the ability of the proposed LED driver to adjust the drive voltage for reducing current imbalance, resistors of 2.2 and $3.9 \Omega$ are added to the second and third LED strings, respectively, so that an exaggerated mismatch situation among the three LED strings is created. Fig. 7 (b) shows the new waveforms under this situation. As expected, the pulse width of $V_{r 3}$ is widest because the third string has the

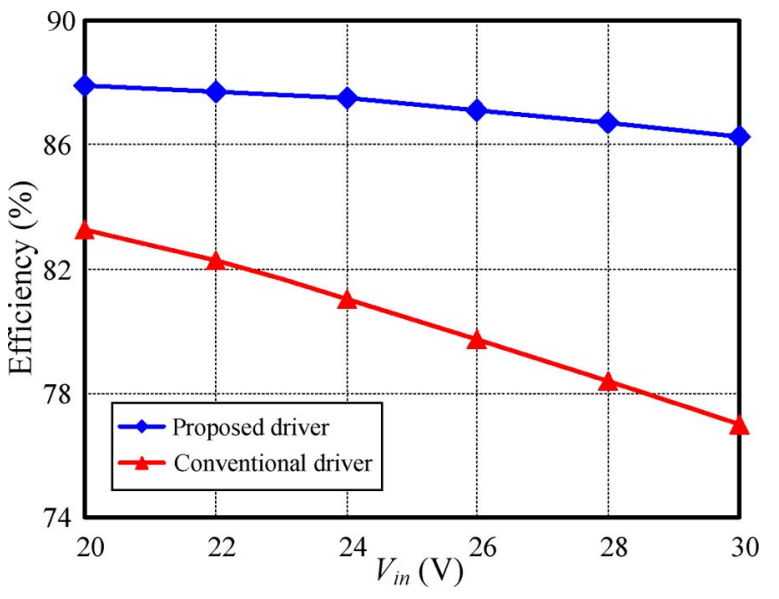

Fig. 10. Efficiency comparison between the proposed driver and the conventional multioutput mag-amp regulated driver

highest extra resistor; the pulse width of $V_{r 2}$ is wider than that of $V_{r 1}$, which remains unchanged. The new measured voltages are $V_{m}=17.06 \mathrm{~V}, V_{s 1}=1.87 \mathrm{~V}, V_{s 2}=2.52 \mathrm{~V}$, and $V_{s 3}=2.94 \mathrm{~V}$.

Fig. 8 shows the measured LED string currents with conventional PWM dimming approach (see Fig. 5) under different duty cycles. Identical amplitude of $300 \mathrm{~mA}$ can be achieved for the 


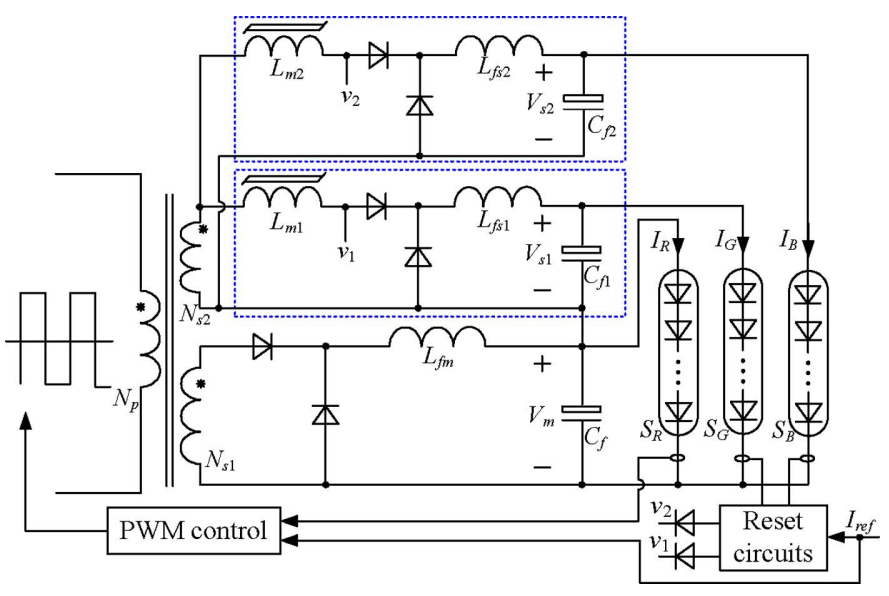

Fig. 11. Circuit diagram of the proposed LED driver for RGB LED strings.

three LED strings under different duty cycles by regulating the voltages of three slave sources, while having only one dimming switch. Fig. 9 shows the waveforms of the proposed LED driver under PSPWM dimming approach (see Fig. 6) under different duty cycles. Again, good current balance has been practically achieved under all these conditions.

A conventional LED mag-amp regulated driver based on [25] is built for comparison purpose. Fig. 10 shows the measured overall efficiency of the proposed LED driver and the conventional one under different input voltages. Due to the use of a common power supply and the relative low-power handling requirements of the mag-amp postregulators, higher energy efficiency has been achieved by the proposed scheme.

\section{APPLICATION TO RGB LED DRIVER}

For LCD backlight application, the RGB LEDs mixing threecolor lights to white light are often employed. It has been point out in [12] that the nominal forward voltages of RGB LEDs are different. The forward voltage of red LED is lower than those of green and blue ones from the same manufacturer, and the forward voltage of green LED is approximate the same as that of the blue one. In the light of these factors, the proposed LED driver is suitable for RGB LED application. The proposed circuit can be used for such application. In Fig. 11, the red LED string is powered by the master source, and the green and blue LED strings are powered by the combination of the master source and corresponding slave sources. The currents of green and blue LED strings are separately regulated by corresponding adaptive slave voltage source for current sharing; however, the current of red LED string is just regulated by the master voltage source for current sharing. Compared with the multiconverter structures [20], the circuit in Fig. 11 is simpler. Compared with multioutput flyback converter [24], each output voltage across LED string is independently regulated to the optimal value, thus leading to higher efficiency.

\section{CONCLUSION}

A dimmable LED driver with mag-amp postregulator for multistring applications has been proposed. A two-secondary- winding structure is proposed in the driver circuit to generate a master source and several slave sources. The master source provides the majority of power for all the LED strings, and the remaining power of each LED string is handled by the corresponding slave source. Each slave source is separately regulated by a mag-amp feedback loop to regulate the LED string forward current. Conventional PWM and PSPWM dimming methods have been successfully implemented in the proposed LED driver. Without linear current regulators, the proposed driver has relatively higher efficiency. Compared with multiconverter structures, the proposed circuit is simpler for RGB LED application. The proposed driver is particularly suitable for highperformance applications. The feasibility and advantageous features of the proposed driver have been practically verified by a prototype.

\section{ACKNOWLEDGMENT}

The authors are grateful to the Centre for Power Electronics, City University of Hong Kong (CityU), for its support for this project. The funding support of CityU for the patent application is also acknowledged.

\section{REFERENCES}

[1] [Online] Available: http://www.colorkinetics. com/support/whitepapers/ LEDLifetime.pdf, 2011.

[2] B. Wang, X. Ruan, K. Yao, and M. Xu, "A method of reducing the peakto-average ratio of LED current for electrolytic capacitor-less AC-DC drivers," IEEE Trans. Power Electron., vol. 25, no. 3, pp. 592-601, Mar. 2010.

[3] S. Y. R. Hui, S. Li, X. Tao, W. Chen, and W. M. Ng, "A novel passive off-line light-emitting diode (LED) driver with long lifetime," in Proc. IEEE Appl. Power Electron. Conf. (APEC), 2010, pp. 594-600.

[4] L. Gu, X. Ruan, M. Xu, and K. Yao, "Means of eliminating electrolytic capacitor in AC/DC power supplies for LED lightings," IEEE Trans. Power Electron., vol. 24, no. 5, pp. 1399-1408, May 2009.

[5] S. Y. R. Hui and Y.X. Qin, "General photo-electro-thermal theory for lightemitting diodes (LED) systems," IEEE Trans. Power Electron., vol. 24 no. 8, pp. 1967-1976, Aug. 2009.

[6] Y. X. Qin and S. Y. R. Hui, "Comparative study on the structural designs of LED devices and systems based on the general photo-electro-therma theory," IEEE Trans. Power Electron., vol. 25, no. 6, pp. 507-513, Feb. 2010.

[7] X. Qu, S.-C. Wong, and C. K. Tse, "Temperature measurement technique for stabilizing the light output of RGB LED lamps," IEEE Trans. Instrum. Meas., vol. 59, no. 3, pp. 661-670, Mar. 2010.

[8] M. Khatib, "Ballast resistor calculation: Current matching in parallel LEDs," Texas Instrument Application Report SLVA325, Apr. 2009.

[9] S. M. Baddela and D. S. Zinger, "Parallel connected LEDs operated at high frequency to improve current sharing," in Proc. IEEE Ind. Appl. Soc. (IAS), 2004, pp. 1677-1681.

[10] H. Chang and Y.-S. Lai, "Novel AC driver and protection circuits with dimming control for light emitting diodes," in Proc. IEEE Ind. Appl. Soc. (IAS), 2007, pp. 696-700.

[11] S. Choi, P. Agarwal, T. Kim, J. Yang, and B. Han, "Symmetric current balancing circuit for multiple DC loads," in Proc. IEEE Appl. Power Electron. Conf. (APEC), 2010, pp. 512-518.

[12] M. Doshi and R. Zane, "Digital architecture for driving large LED arrays with dynamic bus voltage regulation and phase shifted PWM," in Proc. IEEE Appl. Power Electron. Conf. (APEC), 2007, pp. 287-293.

[13] L. Burgyan and F. Prinz, "High efficiency LED driver," U. S. Patent 6690146, Feb. 10, 2004

[14] Y. Hu and M. M. Jovanović, "LED driver with self-adaptive drive voltage," IEEE Trans. Power Electron., vol. 23, no. 6, pp. 3116-3125, Nov. 2008.

[15] H.-J. Chiu, Y.-K. Lo, J.-T. Chen, S.-J. Cheng, C.-Y. Lin, and S.-C. Mou, "A high-efficiency dimmable LED driver for low-power lighting applications," IEEE Trans. Ind. Electron., vol. 57, no. 2, pp. 735-743, Feb. 2010. 
[16] J. Hasan, D. H. Nguyen, and S. Ang, "A RGB-driver for LED display panels," in Proc. IEEE Appl. Power Electron. Conf. (APEC), 2010, pp. 750754.

[17] C.-L. Chiu and K.-H. Chen, "A high accuracy current-balanced control technique for LED backlight," in Proc. IEEE Power Electron. Spec. Conf. (PESC), 2008, pp. 4202-4206.

[18] [Online]. Available: http://cds.linear.com/docs/Datasheet/3452f.pdf. 2006.

[19] H.-J. Chiu and S.-J. Cheng, "LED backlight driving system for large-scale LCD panels," IEEE Trans. Ind. Electron., vol. 54, no. 5, pp. 2751-2760, Oct. 2007.

[20] S. Muthu and J. Gaines, "Red, green and blue LED-based white light source: Implementation challenges and control design," in Proc. IEEE Ind. Appl. Soc. (IAS), 2003, pp. 515-522.

[21] K. I. Hwu and Y. T. Yau, "Applying one-comparator counter-based sampling to current sharing control of multi-channel LED strings," in Proc. IEEE Appl. Power Electron. Conf. (APEC), 2010, pp. 737-742.

[22] Q. Hu and R. Zane, "LED driver circuit with series-input-connected converter cells operating in continuous conduction mode," IEEE Trans. Power Electron., vol. 25, no. 3, pp. 574-582, Mar. 2010.

[23] X. Qu, S.-C. Wong, and C. K. Tse, "Noncascading structure for electronic ballast design for multiple LED lamps with independent brightness control," IEEE Trans. Power Electron., vol. 25, no. 2, pp. 311-340, Feb. 2010.

[24] C.-Y. Wu, T.-F. Wu, J.-R. Tsai, Y.-M. Chen, and C.-C. Chen, "Multistring LED backlight driving system for LCD panels with color sequential display and area control," IEEE Trans. Ind. Electron., vol. 55, no. 10, pp. 3791-3800, Oct. 2008.

[25] C.-C. Chen, C.-Y. Wu, and T.-F. Wu, "Fast transition current-type burstmode dimming control for the LED back-light driving system of LCD TV," in Proc. IEEE Power Electron. Spec. Conf. (PESC), 2006, pp. 29492955.

[26] S.-A. Liang and C.-P. Wu, "A new and simple structure of PC power supply with low cost and high efficiency to meet 80 plus requirement," in Proc. IEEE Appl. Power Electron. Conf. (APEC), 2008, pp. 1347-1352.

[27] P. Degen, H. de Groot, R. Pagano, F. Pansier, and K. Schetters, "A power management unit with integrated stand-by supply for computer motherboards: Control and VLSI design," IEEE Trans. Power Electron., vol. 23, no. 4, pp. 2093-2105, Jul. 2008.

[28] D. Maksimović and R. Erickson, "Modeling of cross-regulation in multiple-output flyback converters," in Proc. IEEE Appl. Power Electron. Conf. ( APEC), 1999, pp. 1066-1072.

[29] A. Barrado, E. Olías, A. Lázaro, J. Pleite, and R. Vázquez, "PWM-PD multiple output DC/DC converters: Pperation and control-loop modeling," IEEE Trans. Power Electron., vol. 19, no. 1, pp. 140-149, Jan. 2004.

[30] Y.-T. Chen and J.-M. Liang, "Paralleling magamp-postregulator modules with sliding-mode-control method," IEEE Trans. Ind. Electron., vol. 53, no. 3, pp. 974-983, Jun. 2006

[31] W.-K. Lun, K. H. Loo, S.-C. Tan, Y. M. Lai, and C. K. Tse, "Bilevel current driving technique for LEDs," IEEE Trans. Power Electron., vol. 24, no. 12, pp. 2920-2932, Dec. 2009.

[32] K. H. Loo, W.-K. Lun, S.-C. Tan, Y. M. Lai, and C. K. Tse, "On driving techniques for LEDs: Toward a generalized methodology," IEEE Trans. Power Electron., vol. 24, no. 12, pp. 2967-2976, Dec. 2009.

[33] C.-C. Chen, C.-Y. Wu, Y.-M. Chen, and T.-F. Wu, "Sequential color LED backlight driving system for LCD panels," IEEE Trans. Power Electron., vol. 22, no. 3, pp. 919-925, May 2007.

[34] Y.-K Lo, K.-H Wu, K.-J Pai, and H.-J Chiu, "Design and implementation of RGB LED drivers for LCD Backlight modules," IEEE Trans. Ind. Electron., vol. 56, no. 12, pp. 4862-4871, Dec. 2009.
[35] Design of High Frequency Mag Amp Regulators Using METGLAS Amorphous Alloy 2714 A, Parsippany, NJ: Allied-Signal Company, 1991.

[36] B. Mammano, "Magnetic amplifier control for simple, low-cost secondary regulation," Unitrode Seminar Manual SEM500, pp. 7.1-7.9, 1986.

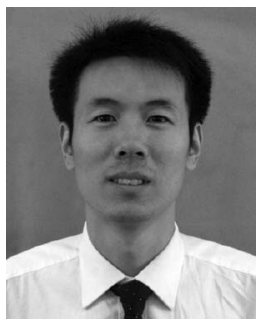

Wu Chen (S'05) was born in Jiangsu, China, in 1981 He received the B.S., M.S., and Ph.D. degrees in electrical engineering from the Nanjing University of Aeronautics and Astronautics, Nanjing, China, in 2003, 2006, and 2009, respectively.

From 2009 to 2010, he was a Senior Research Assistant in the Department of Electronic Engineering, City University of Hong Kong, China. He is currently a Postdoctoral Researcher in Future Renewable Electric Energy Delivery and Management Systems Center, North Carolina State University, Raleigh.

His main research interests include soft-switching dc/dc converters, inverters, and power electronic system integration.

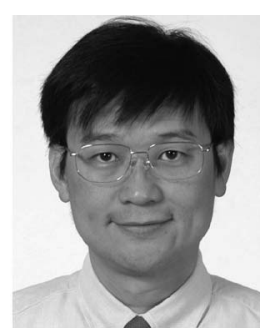

S. Y. R. Hui (F'03) received the B.Sc. Eng. (Hons.) degree from the University of Birmingham, Birmingham, U.K., in 1984, and the D.I.C. and Ph.D. degrees from the Imperial College of Science and Technology, London, U.K., in 1987.

From 1987 to 1990 , he was a Lecturer at the University of Nottingham, U.K. In 1990, he joined the University of Technology, Sydney, and was appointed as a Senior Lecturer at the University of Sydney, in 1992, where he became a Reader in 1995. In 1996 , he joined the City University of Hong Kong (CityU) as a Professor, where he became a Chair Professor in 1998. From 2001 to 2004 , he was an Associate Dean at the Faculty of Science and Engineering at CityU. Since 2010, he holds the Chair Professorship at both CityU and Imperial College London. He is the author or coauthor of more than 200 published technical papers, including more than 140 refereed journal publications and book chapters. Over 45 of his patents have been adopted by industry.

Dr. Hui is a Fellow of the Institution of Engineering and Technology (IET). He has been an Associate Editor (Power Conversion) of the IEEE TRANSACTIONS ON POWER ELECTRONICS since 1997 and an Associate Editor (Lighting Technology) of the IEEE TRANSACTIONS ON INDUSTRIAL ELECTRONICS since 2007. He has been appointed twice as an IEEE Distinguished Lecturer by the IEEE Power Electronics Society in 2004 and 2006. He was one of the 18 Administrative Committee members of the IEEE Power Electronics Society and was the Chairman of its Constitution and Bylaws Committee from 2002 to 2010. He is the recipient of the Excellent Teaching Award at CityU in 1998 and the Earth Champion Award in 2008. He won an IEEE Best Paper Award from the IEEE Industry Applications Society Committee on Production and Applications of Light in 2002, and two IEEE Power Electronics Transactions Prize Paper Awards for his publication in Wireless Battery Charging Platform Technology in 2009 and for his paper on LED system theory in 2010. His inventions underpin key dimensions of Qi the world's first wireless power standard, with freedom of positioning and localized charging features. In Nov. 2010, he received the IEEE Rudolf Chope R\&D Award from the IEEE Industrial Electronics Society, the IET Achievement Medal (The Crompton Medal), and was elected to the Fellowship of the Australian Academy of Technological Sciences and Engineering. 